\title{
Infrastructural Model for the Healthcare System based on Emerging Technologies
}

\author{
Aleksandra Vukmirović ${ }^{1}$, Zoltán Rajnai ${ }^{2}$, Miroslav Radojičić ${ }^{2}$, \\ Jovanka Vukmirović ${ }^{3}$, Marina Jovanović Milenković ${ }^{3}$
}

${ }^{1}$ Belgrade Business School Higher Education School for Applied Studies, Kraljice Marije 73, 11000 Belgrade, Serbia, aleksandra.vukmirovic@bbs.edu.rs

${ }^{2}$ Óbuda University, Bécsi út 96/b, 1034 Budapest, Hungary, rajnai.zoltan@bgk.uni-obuda.hu,dr.mradojicic@gmail.com

${ }^{3}$ Faculty of organizational sciences University of Belgrade, Jove Ilića 154, 11000 Belgrade, Serbia, vukmirovic.jovanka@fon.bg.ac.rs, marinaj@fon.bg.ac.rs

Abstract: Infrastructural model presented and developed in this paper represents a pillar for medical research and has a profound implication in the future of healthcare. The roposed model is based on Big Data concept that refers to technologies and techniques that consist of diverse data that changes rapidly and is too big and therefore cannot be efficiently processed by traditional computer technology and infrastructure. Components of this model are described in detail. In addition, this paper illustrates advantages, limitations, examples and recommendations for the use of Big Data in the healthcare system.

\section{Introduction}

Thorough analysis of large amounts of data that is available, not only for healthcare system, but within the whole society, have shown that there are new possibilities for growth, which are eminent in many scientific disciplines, as well as in medicine [24]. This technological growth leads to a more efficient healthcare system: reduction in costs of treatments - faster and more precise diagnostics, better controlled use of prescribed medication, more effective monitoring of epidemics, pandemics and etc. [3] [5] [48].

The main problem considered in this paper is creation of a model based on emerging technologies and Big Data concept for improvement of the healthcare system. Focus is set on creating a reliable, distributive and scalable infrastructure 
for managing, analysing, sharing, storing and sending large amounts of data. In medical research, available data are both structured and unstructured, stored in various formats: text, pictures, audio, video, clickstreams, log files, sensor files, etc. Data is often time sensitive and needs to be quickly delivered and analysed [17] [43].

The main goal that would be achieved by the implementation of a model developed in this paper is the emphasized importance for the introduction of emerging IT concepts in the process of medical research; which will significantly improve the output performance of healthcare system as a whole. This is primarily related to Big Data technologies and solutions; whose implementation will necessarily start methodological changes in the traditional research process that has been used for standard medical research so far.

Additional goals set in this paper are:

- Development of research methodology, which should lead to improvements in the data collection process by introducing the concept of Big Data, regarding timeliness, uniqueness and comprehensiveness of information related to medical research.

- Implementation of services and applications for medical research based on modern information technology,

- Optimal use of available IT resources,

- $\quad$ Pointing out the directions of further development in this area - especially in the field of research methodology and domain analytics.

This paper presents the benefits of implementing Big Data analytics in the field of medicine, the impact that emerging technologies have on preventing diseases, and describes examples reported in the literature [42]. The most important contribution of this paper is reflected in development of the infrastructure model based on new methodological assumptions. This model has solved problems such as collecting and storing large amounts of data, which creates conditions for their further analysis and presentation.

The main hypothesis that will be tested in this paper is: there are large amounts of available data (both structured and unstructured), from various sources that are not directly related to the healthcare system, but could be used for medical research.

The main hypothesis can be divided into two specific hypotheses:

H0.1. Emergence and implementation of Big Data necessarily leads to a change in the traditional methodology of processing, analysis and presenting data, and overall conducting medical research.

H0.2. Traditional statistical methods in the field of statistical inference (hypothesis testing) inevitably must adapt to the use of large amounts of data that are collected continuously in real-time. 
The research methods used in developing this model are based on the existing theoretical approaches and on experimental work in this scientific field. Accordingly, scientific literature was consulted and referenced during the analysis phase of the model's content and systematization of knowledge. Implementation of the developed model should confirm the main hypothesis. The research methods implemented in this paper are data analysis methods: comparative and modelling methods.

\section{The Importance of Emerging Technologies in Medicine}

This paper provides a broad overview of Big Data technology for healthcare researchers and practitioners. The results of this research contribute to the accuracy of requirements necessary for the implementation of infrastructure in medical research [42].

Big Data technologies can be defined as datasets that are too large for traditional data-processing systems and thus require new technologies [39] [41]. In accordance with this general definition, Big Data can furthermore be defined as a set of data that overcomes the capabilities of typical software for databases management in the health care - data collection, storage, managing and analysing large amounts of data [7].

Big Data refers to the amount of data that is being produced on a daily basis, and describes the limits of existing data storages and computer power. It is a known fact that the healthcare system generates large amounts of data. Modern information technology has dramatically changed the landscape of how healthcare is delivered and studied. Per experts' estimation the worldwide digital healthcare data reached was 25,000 petabytes in 2014. According to the report of the Institute for Health Technology Transformation the U.S. healthcare system reached was 150 exabytes in 2011. It is estimated that at this growth rate, Big Data of the U.S. healthcare system will soon reach the petabyte scale and not long after, the yottabyte scale [42].

Aside from the volume, the Big Data concept is characterized by its velocity for information processing in real-time. It primarily assumes the time necessary to obtain a final result, which calls for action. The reasons for the imperative of the velocity in the healthcare are [15]:

- the very nature of medical activities, such as: the occurrence of an epidemic, diagnosis, response to therapy, the prognostics, etc. 
- the medical data have short expiration dates and very quickly become obsolete due to the changes in the environment and the health status of patients.

In order to gain insight into the essence of the data as soon as possible all of the above facts confirm that the data must be collected, processed and analysed in real time. This also implies that the paradigm of the medical research is changing: data needs to be analysed from the moment of its collection [9] [44]. This means that the process of data collection is continuous in real time, because prompt actions are necessary in medicine, more than in any other field [6].

Big Data concept is based on another important characteristic - variety of data [42]. The development of information technology has led to the emergence of different types of data that could be collected [10] [11]. The structured data by simplest definition is all data collected, stored and presented by traditional methods, while all other data are defined as unstructured data. Big Data concept is based on the use of large quantities of unstructured data. When it comes to health care, these data sources can be internal (within the health institution) and external (coming from the environment).

The most common Internal unstructured data generated in the health care system are [23] [42]: Text (paper documents or electronic records: office medical records, handwritten nurse and doctor notes, hospital admission and discharge records, paper prescriptions, etc.), Medical Imaging Data (2D and 3D, x-ray films, MRI, CT and other images). Structural data consists of genetic data and data that represent regular monitoring, for example: multiple daily diabetic glucose measurements, blood pressure readings, EKGs, etc.

\section{Internal and external structured data}

Structural data in healthcare can be external and internal. External structured data comes from the environment while internal data is produced within the healthcare information system or clinical decision support systems. The electronic healthcare documentation in ideal conditions presents information about the patient since its birth and it is being collected from numerous healthcare institutions [25]. There are three major types of structured and semi-structured data in the healthcare sector as follows [14] [37]:

- Clinical data (electronic medical records, digital images, and informationsensing wireless medical devices),

- Various Medical research records,

- Business/organization operations records (data from administrative sources: financial, technical, etc.).

The structured data in electronic medical records includes: basic information about the patient (name, date of birth), physician's name, patient medical history, insurance information and any other information that is stored in existing 
databases. As illustrated in the Figure 1, structured data is data that can be easily stored, queried, recalled, analysed and manipulated. This figure represents electronic healthcare documentation inside the healthcare system based on the example of the Republic of Serbia. Usually structured and semi-structured data in healthcare include instrument readings and data generated by the ongoing conversions from paper records into electronic health and medical records [10].

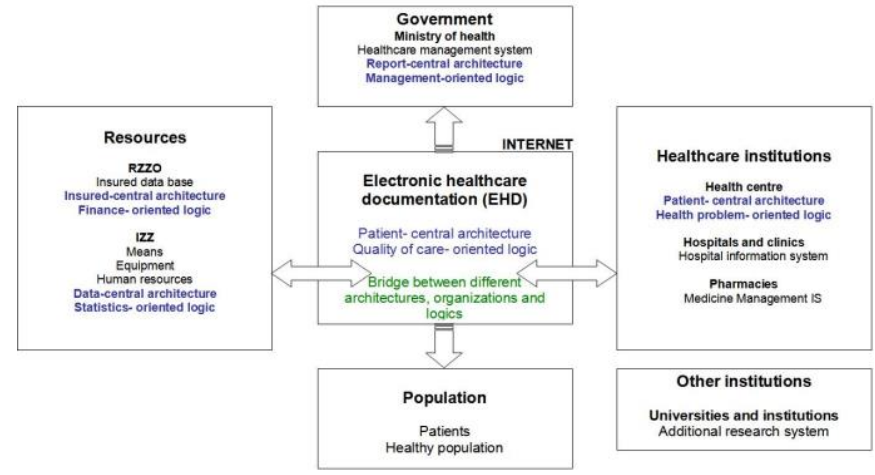

Figure 1

Electronic Healthcare Documentation inside the Healthcare System in Serbia [33]

A certain number of structured data exists in the external environment, such as data that is based on transactions in the field of insurance, health services, financial transactions, communications, etc. Accordingly, proposed model uses the data that is not essentially medical, but covers demographic, economic and lifestyle variables of individuals - patients. It is the use of new "medical" data that truly separates the proposed model from traditional solutions. This data is combined with the analysis of "classic" health data to improve health services, especially for prevention and diagnosis.

External unstructured data is being generated outside the healthcare system. Those are mostly nonmedical data such as data about demographic, financial and life style variables. External unstructured data are formatted in various forms, where the most common are text, figures, music and video.

There are many factors which are contributing to the volume increase of external unstructured data that becomes relevant to healthcare [29]:

- $\quad$ The increasing use of smartphones and specialized services, such as health applications, personal physical fitness tracking tools, GPS device, bar code reader, and QR reader.

- The number of unstructured data coming from the social media: social networks, 
- Increased amounts of data gathered from reading sensors and similar devices like cameras, climate sensors, and chair and floor sensors.

- $\quad$ Extensive use of cloud technologies.

- The internet of the things concept - the introduction of smart devices in homes and smart meters that are based on Bluetooth technologies

Ericson company states in its annual Mobility report that in December 2015 there were as many mobile subscriptions as there were people in the world. Ericson estimates that by 2021, the increase of smartphone subscribers will double, considering the fact that in the third quarter of 2015 nearly $75 \%$ of mobile phones sold worldwide were smartphones. [12].

McKinsley \& Co. acknowledged that the IoT (Internet of things) applications for health and fitness are very well adopted by the smartphone users. Based on current number of users of smart devices (phones, tablets, watches, wristbands, etc.) and projected growth of that number in the future, it is estimated that the economic income from the sold IoT applications for health and fitness will be between $\$ 170$ billion to nearly $\$ 1.6$ trillion globally in 2025 . The largest source of value would be use of IoT devices for monitoring and treating illness [32]. Value would arise from improving quality of life and extending healthy life spans for patients with chronic illnesses, and reducing cost of treatment. Emerging applications have the potential to transform a wide range of health-care therapies. Ingestible and injectable - smart pills and nanobots - have the potential to replace many surgical interventions with less invasive approach that results in reduced medical costs, fast recovery and improved satisfaction of the patient [15].

Investments in IoT systems are proven to be justified, especially in the field of disease prevention, early diagnostics and cost reduction. One of the determinants that can be measured with more accuracy by the smart devices than with a physician appointment are the changes of human behaviour (for example, mood changes, routine changes, sleeping disorders, etc.) [28]. Often before the worsening of a patient's condition there is a period in which physiological data that now can be collected by smartphones, can also be used to determine whether patient is at risk for de-compensating. [2]

\section{Model of Big Data Infrastructure as a Pillar of Technical Support for Medical Research}

In a regular health analytics project, the analysis can be performed with a business intelligence tool installed on a stand-alone system, such as a desktop or laptop. Since Big Data is large by definition, processing is broken down and executed across multiple nodes. The concept of distributed processing has existed for 
decades. What is relatively new is its use in analysing very large data sets as healthcare providers start to tap into their large data repositories to gain insight for making better-informed health-related decisions. [20] [35]

Especially, in case of science Big Data, scientists tend to move Big Data from the origin site of the Big Data to the nearest data centre because it is hard for scientists to analyse data remotely due to the long delay in time that occurs in the read/write process. After moving Big Data to the nearest data centre, scientists analyse it with thousands of CPUs that are connected by a very high-speed local network in the data centre. [38]

Open source platforms as Hadoop and MapReduce [49], available on the cloud, have encouraged the application of Big Data analytics in healthcare.

Suggested infrastructural model developed in this paper (Figure 2) cannot be integrated with existing management solutions (information system, CRM applications, etc.) primarily due to the use of various definitions, and thus it was necessary to start modelling from the beginning.

Implementing the developed model of Big Data infrastructure for technical support in medical research (Figure 2) will lead to the real-time integrated management of the information. The main characteristics of this model are (Figure 2):

- Data that has been analysed are external secondary data,

- Collected data are poorly structured (located somewhere outside the organization and are not suitable for analysis without further processing),

- Model is not constructed to analyse past situations, but to predict the future.

Basic infrastructure model layers, shown in Figure 3 are the layers of software infrastructure and the layers of hardware infrastructure.

Software infrastructure component is based on the use of the concept of Big Data and Cloud Computing solutions, applied to the process of medical research. All software applications and services are implemented on the Cloud infrastructure [36].

Figure 4 illustrates the cyclical character of the developed model that enables one of the most important requirements of its design - reporting in real time. 


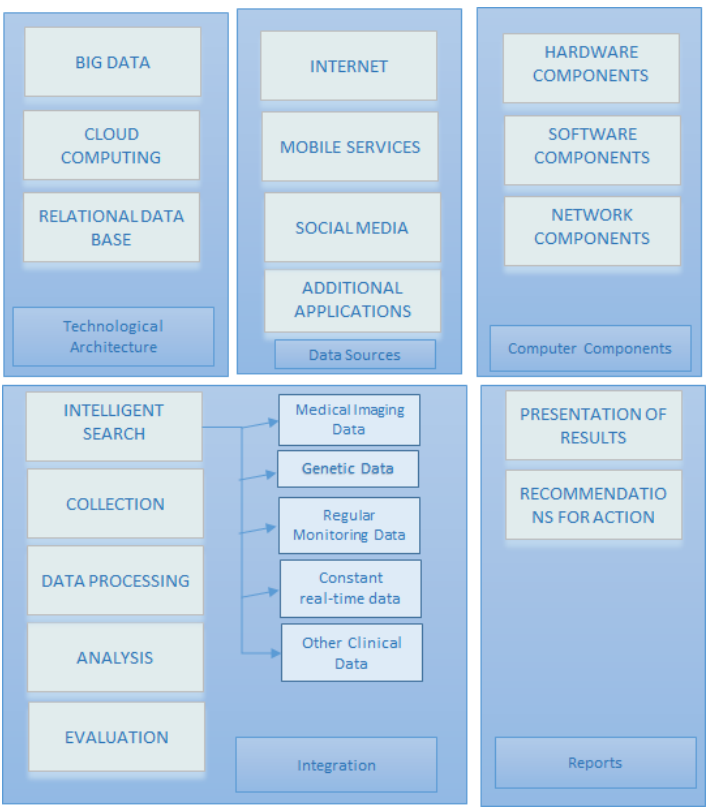

Figure 2

Developed general model of Big Data infrastructure for medical research technical support
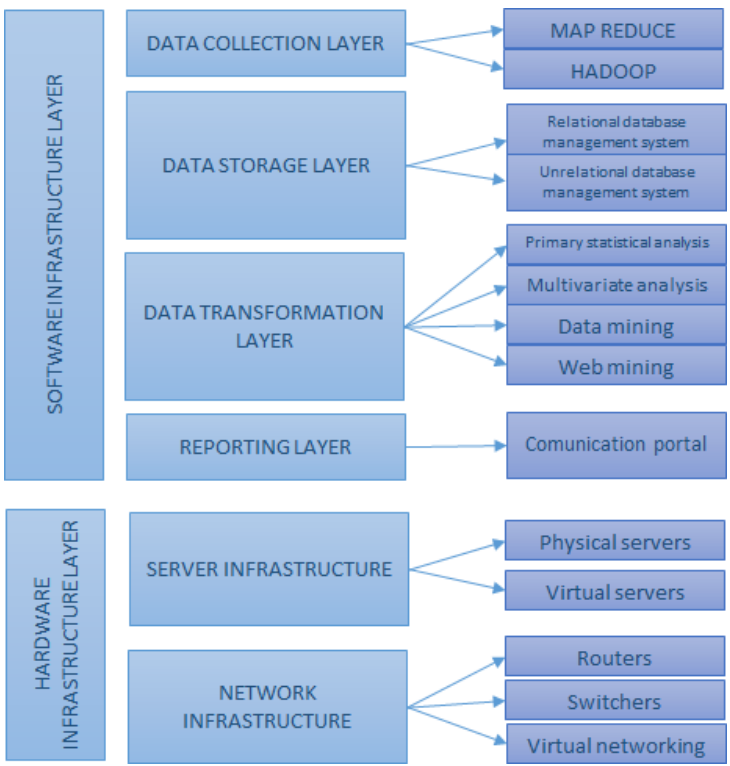

Figure 3

Multilayer infrastructural model 


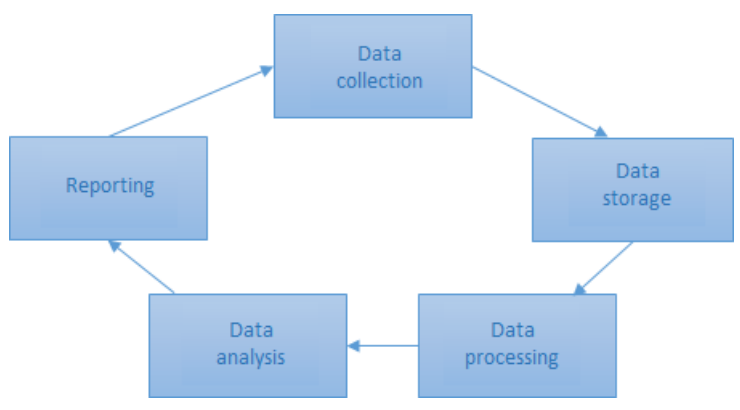

Figure 4

Cycle infrastructural model

Thanks to the "concept of a cycle" of this model and its dynamics, evaluation of results are being conducted automatically. Practically, every newly entered data entry is changing a previous report. This way, it meets the basic requirement of users - the real-time reporting.

From the standpoint of the infrastructure of the model, reporting component is being developed as the reporting portal consisted of two parts: Module for presenting the results and Module for forecasting.

Both modules are mutually connected and dynamically updated - as new data arrives in the model it is being stored, processed and analysed.

\section{Discussion}

In the previous sections of this paper, a model of Big Data infrastructure that can be used for medical research has been developed. This model generates and integrates various types of data that may have an impact on a person's health (demographic, financial, life style, etc.) and sends that data for analysis and reporting. The model opens the possibility for different devices to send data into the cloud which are then combined with different data and processed into medical information in real time. It is particularly important that this data can be combined with data from medical records, which offers the possibility of introducing new variables and measuring their impact in medical research. Further research will attempt to show how the model works in the case of establishing the early dementia.

Data will be collected from various sources provided by smart devices, mainly smartphones. Participants will be monitored in in-house and out-house environment. Collected data will be stored on the cloud server where it will be processed and paired with the existing EMRs (Electronic Medical Records) of the participants using Big Data technologies and Hadoop open source software [26]. 
Main data source is health application that collects: activity data using GPS and Bluetooth technologies (automatically), caffeine intake (by input), water intake (by input), heartbeat (measurement by the sensor implemented into the smartphone), nutrition (by input), sleep duration (automatically), stress amount (measurement by the sensor implemented into the smartphone). Data will be collected from other various mobile applications such as weather application (temperature, humidity, air pressure, UV index, noise level), and for female participant's period applications, agenda entry - in order to monitor the participants' focus on remembering daily tasks.

Monitoring the call logs, WhatsApp, Viber and messaging will provide data about socialization and human interaction, while microphone is being used for detecting voice changes. [51] In in-door conditions data can be collected by various sensors that monitor the participant's movement and position: intelligent chair sensor that detects posture [31], floor sensors for detecting falls [27], door sensors for detecting movement around home, out-house environment: For research purposes and maximizing of data collection, research will be conducted in urban environment. This data should represent the life style of the participants in outside environment. Primarily, the data collected using a smart phone that monitors their movement and activity (health applications + GPS) over hot-spots covered areas. Depending on the smart city development, the data can be combined with public transportation data (data provided by subway and/or bus cards).

Available data applicable in this research are credit card statements and list of groceries ordered online. Also, participants will be encouraged to scan the bills when shopping with the aim to identify a set of variables relating to food and financial status of the participants. Missing data that cannot be collected automatically using emerging technologies can be entered by participants on a daily basis through interactive questionnaires and reminders.

Most of the data that is collected from in-house and out-house environment are external unstructured data (data generated outside the healthcare system). The proposed infrastructure model allows this data to be collected and stored. This presents an opportunity for combining external with internal data (structured and unstructured), which is also stored in the same system and has already been collected within the health institution that takes care of the patient - research participant. Based on the database, healthcare institutions and care managers will monitor the patients' data. Real-time reporting is just one important future use of Big Data. Reporting may be displaying current results and forecasts. [12]

Until now, there could not be any forecasts analytics in healthcare because there was no data available. Applying this model of Big Data in healthcare fills-in the gap. Socioeconomic factors are an example in predictive analytics. This and similar data can help organizations to establish the exact diagnosis for the patient. Using this model, the report provides an analysis of data, noncompliance with medications, and more. That is just a small example of how Big Data can fuel predictive analytics. [18] 
These predictive analytics predicts the state of health of a patient. Based on data from other patients with similar conditions, predictive algorithms can predict the state of health of patients over the time. [40]

Big Data technologies have the potential to transform the way healthcare providers use sophisticated technologies to gain insight from their clinical and other data repositories and make decisions based on collected information. In the future, we will see rapid, widespread implementation and use of Big Data analytics across the healthcare organization and the healthcare industry [42]. To that end, the several challenges highlighted above, must be addressed. As Big Data analytics becomes more mainstream, issues such as guaranteeing privacy, safeguarding security, establishing standards and governance, and continually improvement of the tools and technologies will garner attention. [2] Big Data analytics and applications in healthcare are at a nascent stage of development, but rapid advances in platforms and tools can accelerate their maturing process.

An additional challenge is the lack of experts - data scientists, experts not only in IT technologies, but also in the field of statistics, data mining and health statistics. It is important to emphasize that the research methodology and conclusion based on Big Data concept (and the proposed infrastructure model) must be developed in this field to ensure the verification of the results obtained on a scientific basis.

The benefits from implementing emerging technologies as a pillar of the healthcare system have been recognized by organisations world-wide. The USA National centre for scientific research has developed a project named City4Age that presents Ambient Assisted Cities for supporting older people with mild cognitive impairment to live independently. By collecting frequent data on behaviours, this project will alleviate the role of health services for older people that are at risk or have mild cognitive impairment. [45]

IBM and Microsoft have declared the partnership in developing the healthcare system based on Big Data technologies allowing iPhone and Apple Watch users to share the data from their devices to IBM's Watson Health cloud-based healthcare analytics service. [22]

University of Iowa is predicting that by year 2020, the amount of data available in healthcare will double every 73 days. [46] Therefore, the introduction of Big Data model into the health system is inevitable and has a great potential in improving healthcare.

\section{Conclusions}

This paper presents a comparative analysis of the possibilities and limitations of new technologies (ICT) applied in the healthcare system. The paper shows that this field of the medicine cannot remain on the side-lines when it comes to implementing new IT concepts, especially Big Data concept. Emerging ICT technologies have a direct impact on changing the current practice in the field of all types of research, including medical. In concrete terms, Big Data is not only 
new technology; it is a new approach to medicine that requires improvement of research methodology, which has so far largely relied on classical statistical methods of inference.

Suggested model presents the first step in implementing this concept of adapting modern data warehousing solution to the concept of large amount of data. In the case of detection of dementia, benefits of technology developments were illustrated - enormous amount and variety of data is generated, but not yet fully available for implementation in healthcare. We pointed out the sustainability of the base hypothesis.

The main hypothesis tested in this paper: there are large amounts of available data (structured and unstructured) from various sources that can be used for medical purposes which are not directly related to the health system - was confirmed.

Also, first specific hypotheses was proven: Emergence and implementation of Big Data necessarily lead to a change in the traditional methodology of processing, analysis and presenting data, and overall conducting medical research. In addition to the manner of collection, the challenge is in processing and analysing the collected data. The second specific hypothesis goes beyond information frames and has yet to be demonstrated in future operations, primarily by statisticians, and data scientists.

The application of this model for medical purposes in Serbia is inevitable. In the past few years, informational scientists in Serbia have been working on introducing informational systems in healthcare facilities. However, the initial situational analysis shows that including Big Data technologies is essential for the growth of healthcare informational system. Introducing Bid Data technologies would present a qualitative leap in the effective use of information for the benefit of the patient and process management in the healthcare system.

Serbian Ministry of Health has announced that during the year 2016, healthcare will revitalize itself with a unique data centralization information system. However, without conducting the findings and knowledge that has been apprehended all around the world by applying Big Data technologies in the healthcare field, the results will be poor and the benefits negligible.

\section{Further recommendation:}

- Essentially educate medical experts about the importance of introducing new ICT, especially Big Data technologies into medical practice

- It is necessary to make and adopt a strategic and legal framework as a support system for Big Data technologies implementation in Healthcare that will provide a solution for overcoming problems of access and use of personal data by updating privacy and data ownership policies in a way to protect patients' rights and allowing the full potential of data collection and analysis. 
- It is recommended for all states to adopt the form of academic-public-private partnerships, following the example of United Kingdom in 2012. [50]

- Adapting the curricula at universities, collages, and faculties in order to educate students about sciences in data field.

\section{References}

[1] Barnett, M. L., Christakis, N. A., O’Malley, J., Onnela, J. P., Keating, N. L., Landon, B. E.: Physician patient-sharing networks and the cost and intensity of care in US hospitals. Med Care 2012; 50(2):152-60 (2012)

[2] Bates, D. W., Saria, S., Ohno-Machado, L., Shah, A., Escobar, G.: Big Data in Health Care: Using analytics to identify and manage high-risk and highcost patients. Health Affairs, 33, no. 7 (2014)

[3] Beyer, M.A., Douglas, L.: The Importance of 'Big Data': A Definition. Available at: URL: http://www.gartner.com/it-glossary/big-data/ (2012)

[4] Bollier, D.: The Promise and Peril of Big Data. The Aspen Institute (2010)

[5] Bradley, P.: Implications of big data analytics of population health management. Big Data; 1(3): 152-159, DOI: 10.1089/big.2013.0019 (2013)

[6] Bram, J. T., Warwick-Clark, B., Obeysekare, E., Khanjan, M.: Utilization and Monetization of Healthcare Data in Developing Countries. Big Data 2015; 3(2), DOI: 10.1089/big.2014.005 (2015)

[7] Brown, B., Chui, M., Manyika, J.: Are you ready for the era of 'big data'? McKinsley Global Institute (2011)

[8] Csubák, D., Szücs, K., Vörös, P., Kiss, A.: Big Data Testbed for Network Attack Detection. Acta Polytechnica Hungarica; Vol. 13, No. 2, DOI: 10.12700/APH.13.2.2016.2.3 (2016)

[9] Davenport, T.: Big data @ work. Harvard Business School Publishing Corporation (2014)

[10] Devdzic, V., Chaloub-Johansson, C., Weinhara, M.: Development of Health Information System for Basic Health and Pharmaceutical Services in Serbia. European Health Group, Copenhagen, Denmark (2008)

[11] Dobrota, M., Jeremic, V., Markovic, A.: A new perspective on the ICT Development Index. Information Development 28(4): 271-280 (2012)

[12] Ericsson Mobility Report November 2015. Available at: http://www.ericsson.com/res/docs/2015/mobility-report/ericsson-mobilityreport-nov-2015.pdf

[13] Foster P, Fawcett T. What you need to know about data mining and dataanalytic thinking. O'Reilly Media, Inc. (2013)

[14] Frost \& Sullivan. Drowning in Big Data? Reducing Information Technology Complexities and Costs for Healthcare Organizations. 
Available at: URL: http://www.emc.com/collateral/analyst-reports/frostsullivan-reducing-information-technology-complexities-ar.pdf (2010)

[15] Governemets Health - IT Staff. Is IoT potential understated? 2015. Available at: http://www.govhealthit.com/news/iot-potential-understated (2015)

[16] Health Affairs. Big Data and New Knowledge in Medicine: The Thinking, Training, And Tools Needed For a Learning Health System; Available at: http://content.healthaffairs.org/content/33/7/1163.full.pdf+html (2014)

[17] Heart, T., Kalderon, E.: Older adults: are they ready to adopt health-related ICT. International Journal of Medical Informatics. 82(11): 209-231 (2013)

[18] Herland M., Khoshgoftaar TM., Wald, R.: A review of data mining using big data in health informatics. Big Data (2014)

[19] Hersh, W., Jacko, J. A., Greenes, R., Tan, T., Janies, D., Embi, P. J., Payne, P.R.O.: Health-care hit or miss? The Ohio State University, College of Medicine; Nature 470, 327-329, DOI: 10.1038/470327a (2011)

[20] Holmes, A.: Hadoop in practice. Manning Publications Co. (2012)

[21] Huangb, T., Lanc, L., Fanga, X., Ana, P., Mind, J., Wanga, F.: Promises and Challenges of Big Data Computing in Health Sciences. Big Data Research (2015)

[22] IBM Watson Health (C2015 International Business Machines Corporation. Available at: http://www-

05.ibm.com/no/businessconnect/assets/files/IBMWatson-hjelper-leger-tabeslutninger-StefanOhlsson.pdf (2015)

[23] Jimeng, S., Chandan, K. R.: Big data analytics for healthcare. Proceedings of the 19th ACM SIGKDD international conference on Knowledge discovery and data mining; 1525-1525. DOI:10.1145/2487575.2506178 (2013)

[24] Jovanovic Milenkovic, M., Jeremic, V., Martic, M.: Sustainable development in the e-health sector of the European Union. Journal of Environmental Protection and Ecology. 15(1): 248-256 (2014)

[25] Jovanović Milenković, M., Radojičić, Z., Milenković, D., Vukmirović, D.: Applying electronic documents in development of the healthcare information system in the Republic of Serbia. Computer science and informational systems; 6(2), 111-126. DOI: 10.2298/csis0902111J (2009)

[26] Lin, W., Dou, W., Zhou, Z., Liu, C.: A Cloud-based Framework for Homediagnosis Service over Big Medical Data. The Journal of Systems and Software (2014)

[27] Lustrek, M., Gjoreski, H., Kozina, S., Cvetkovi, B., Mirchevska, V., Gams, M.: Detecting Falls with Location Sensors and Accelerometers. 
Proceedings of the Twenty-Third Innovative Applications of Artificial Intelligence Conference (2011)

[28] Manyika, J., Chui, M., Bisson, P., Woetzel, J., Dobbs, R., Bughin, J., Aharon, D.: Unlocking the potential of the Internet of Things. McKinsley \& Company; Available http://www.mckinsey.com/insights/business_technology/the_internet_of_th ings_the_value_of_digitizing_the_physical_world (2015)

[29] Manyika, J., Chui, M., Bisson, P., Woetzel, J., Dobbs, R., Bughin, J., Aharon, D.: Unlocking the potential of the Internet of Things. McKinsley \& Company; Available at: http://www.mckinsey.com/insights/business_technology/the_internet_of_th ings_the_value_of_digitizing_the_physical_world (2015)

[30] Manyika, J., Chui, M., Brown, B., Bughin, J., Dobbs, R., Roxburgh, C., Hung Byers, A.: Big data: The next frontier for innovation, competition, and productivity. McKinsley \& Company (2011)

[31] Martins, L., Lucena, R., Belo, J., Santos, M., Quaresma, C., Jesus, A.P., Vieira, P.: Intelligent Chair Sensor. Engineering Applications of Neural Networks (2013)

[32] McKinsey \& Company. Internet of Things \& Healthcare: two important reports; Available at: http://medgizmo.info/news/internet-of-thingshealthcare-two-important-reports (2015)

[33] Milenković, D., Jovanović Milenković, M., Vujin, M., Aleksić, A., Radojičić, Z.: Electronic health system - development and implementation into the health system of the Republic of Serbia. Vojnosanit Pregl 2012; 69(10): 880-890 (2012)

[34] Milenkovic, N., Vukmirovic, J., Bulajic, M., Radojicic, Z.: A multivariate approach in measuring socioeconomic development of MENA countries. Economic Modelling; 38(C): 604-608 (2014)

[35] Miner, D., Shook, A.: MapReduce design patterns. O'Reilly Media, Inc. (2012)

[36] Narayanan, A., Greco, M., Powell, H., Coleman, L.: The reliability of Big "patient satisfaction" Data. Big Data; 1(3), 141-151, DOI: 10.1089/big.2013.0021 (2013)

[37] Ozminkowski, R., Wells, T., Hawkins, K., Bhattarai, G., Martel, C., Yeh, C.: Big Data, Little Data, and Care Coordination for Medicare Beneficiaries with Medigap Coverage. Big Data; 3(2). DOI: 10.1089/big.2014.0034 (2015)

[38] Park, H. W., Yeo, I. Y., Lee, J. R., Jang, H.: Study on Network Architecture of Big Data Centre for the Efficient Control of Huge Data Traffic. 
Computer Science and Information Systems, Vol. 11, No. 3, 1113-1126 (2014)

[39] Pentland, A., Reid, T., Heibeck, T.: Big data and health, Revolutionizing medicine and Public Health. World Innovation Summit for Health (WISH), Big Data and Health Report. Available at: http://wish-qatar.org/bigdata/big-data (2013)

[40] Poucke, S. V., Thomeer, M., Hadzic, A.: 2015, big data in healthcare: for whom the bell tolls? Critical Care (2015)

[41] Power, D.: Ask Dan! About DSS; Available at: http://dssresources.com/faq/index.php?action=artikel\&id=263 (2014)

[42] Raghupathi, W., Raghupathi, V.: Big data analytics in healthcare: promise and potential. Health Information Science and Systems, 2(3), DOI: 10.1186/2047-2501-2-3 (2014)

[43] Seke, K., Petrovic, N., Jeremic, V., Vukmirovic, J., Kilibarda, B., Martic, M.: Sustainable development and public health: Rating European countries. BMC Public Health. 13(77), 1-7. DOI: 10.1186/1471-2458-13-77 (2013)

[44] Simon, P.: Too Big to ignore - the business case for big data. John Wiley \& Sons, Inc. (2013)

[45] The National Centre For Scientific Research: Elderly-friendly city services for active and healthy ageing (2016) Avaiable at: http://www.ipal.cnrs.fr/project/eu-h2020-city4age

[46] University of Iowa Carver Collage of Medicine: MD Curriculum Renewal (2015) Avaiable at: http://www.healthcare.uiowa.edu/2020/

[47] Vukmirovic, A., Brbaklic Tepavac, M., Vukmirovic, J.: Virtual community research. XX Yu Info Conference Proceedings of the Association for Information Systems and Computer Networks; Kopaonik, Serbia (2014)

[48] Wang, W., Krishnan, E.: Big Data and Clinicians: A Review on the State of the Science. JMIR Medical Informatics, 2(1), doi:10.2196/medinform.2913 (2014)

[49] White, T. Hadoop - The definitive guide. O’Reilly Media Inc. (2012)

[50] Williams, L.: The power of information: Putting all of us in control of the health and care information we need, Department of Health, Avaiable at: https://data.gov.uk/sites/default/files/DH\%20Open\%20Data\%20Strategy_1 0.pdf (2012)

[51] Yanga, J., Lic, J., Mulderd, J., Wange, Y., Chenf, S., Wug, H., Wangb, Q., Panf, H.: Emerging information technologies for enhanced healthcare. Computers in Industry (2015) 